\title{
The Impact of Smoking on Semen Quality and ICSI Outcome in the Obese Men
} Salha Abd El Fatah Saad*1, Nehal Ali Abu-Elnaga ${ }^{1}$, Amira Badereldin Abdel Ghany ${ }^{2}$, Ahmed Fathy Alsherbiny Hassan ${ }^{3}$

${ }^{1}$ Department of Embryology, Zoology Department, Faculty of Science, Al-Azhar University, Egypt.

${ }^{2}$ Department of Embryology, International Islamic Center for Population Studies and Research, Al-Azhar University.

${ }^{3}$ Department of Fertility, International Islamic Center for Population Studies and Research, Assisted Reproductive Unit, Al-Azhar University

*Corresponding author: Salha Abd El Fatah Saad, Mobile: (+20) 01015317144, E-Mail: dr.salhamansoura@gmail.com

\begin{abstract}
Background: Male obesity with or without smoking and raised male BMI can markedly harm quality embryo blastocyst development, clinical pregnancy rate, and live birth rate in couples who underwent in vitro fertilization (IVF) or intracytoplasmic sperm injection (ICSI). Male BMI could be an essential factor influencing IVF-ICSI outcome. Objective: This study aimed to investigate the effect of smoking on semen quality, fertilization, embryo quality, and their impact on pregnancy after ICSI in overweight men. Material and methods: The study was performed on 150 cases in the assisted reproductive unit in International Islamic Center for Population Studies and Research, Al-Azhar University. The recruited males were divided into three groups (50/group): Normal control (C), Overweight (OW), and overweight with a smoking (OW\&S). Their physiological, biochemical, and semen parameters were investigated for all cases. The fertilization rate, cleavage rate, embryo quality, and pregnancy rate were examined and recorded during and after the ICSI process. Results: Percent of abnormal sperm morphology in the OW\&S group showed a very highly significant increase $(\mathrm{P}<0.01)$ than the control group. However, for the overweight with smoking $(\mathrm{OW} \& \mathrm{~S})$ and overweight groups (OW), the percentage of the fertilization rate and the high-quality embryos were significantly lower than the values of the control group $(\mathrm{P}<0.01)$. The percentage of the low-quality embryo was higher in OW\&S and OW groups than in the control group. The lowest rate of positive pregnancy test recorded in overweight with smoking (46\%) followed by overweight $(60 \%)$. Conclusion: the semen parameters and hormonal profile, fertilization rate, embryo quality, and pregnancy rate were significantly lower in smoker men suffering from overweight.
\end{abstract}

Keywords: ICSI; Overweight; Semen quality; Smoking.

\section{INTRODUCTION}

Sperm participation in embryogenesis is done through the haploid genome. Sperms are involved in a variety of processes, including syngamy, cleavage, and epigenetic control, in addition to their genetic content. Infertility affects around 15\% of reproductive-age couples. Twenty-five percent of them can be described by male factors alone and up to $50 \%$ by the combination of female and male factors. Along with genetic and epigenetic abnormalities, other influential factors may contribute to idiopathic male infertility and affect in vitro fertilization (IVF) outcomes ${ }^{(\mathbf{1})}$. Many infertile men have an abnormal semen analysis, whose etiology is often poorly described. These may include environmental, diet, medical, genetic and physiological factors ${ }^{(2)}$.

Throughout fertilization, sperms that have high quality and have healthy chromatin are naturally selected. In assisted reproductive techniques (ART), especially in intracytoplasmic sperm injection (ICSI), sperm that have a DNA breakage are naturally excluded and not selected, and this can affect the fertilization ratio, embryo quality, and implantation rate ${ }^{(3-5)}$.

The impact of paternal obesity on prenatal development was neglected until recently. More than half of males of reproductive age are overweight, according to recent research, and this has a variety of consequences for their children's health ${ }^{(6)}$.
Obesity, defined by the World Health Organization (WHO) as a body mass index (BMI) $\geq 30$ $\mathrm{kg} / \mathrm{m}^{2}$, is a medical condition of excess body fat negatively influencing morbidity and mortality via noncommunicable disease risks ${ }^{(7,8)}$. Although the effect of paternal BMI on sperm function has been studied ${ }^{(9,10)}$, there are few studies on the effects on the paternal health of the fetus and live birth outcomes ${ }^{(11,12)}$.

Attention to human reproduction is how lifestyle factors such as cigarette smoking impacts IVF outcome ${ }^{(13)}$. Cigarette smoke contains cotinine, cadmium, metabolites of nicotine that are toxic, mutagenic chemicals and carcinogens. These chemicals cross the blood-testis barrier. The proof of this correlation is the concentration of toxic materials in seminal plasma as those in the serum; this provides an abnormal environment during spermatogenesis ${ }^{(\mathbf{1 4})}$.

This unnatural environment causes oxidative DNA damage in spermatozoa due to its high content of oxidants ${ }^{(15)}$. This study aimed to investigate the effect of male overweight with or without smoking on semen quality, fertilization, embryo quality and their impact on pregnancy after intracytoplasmic sperm injection (ICSI) outcome.

\section{PATIENTS AND METHODS}

The study population consisted of 150 couples; the men were divided into three groups: 
Control group (C): 50 normal men; 50 overweight $(\mathrm{OW})$, and 50 smokers overweight groups. The study was done in the assisted reproductive unit, International Islamic Center for Population Studies and Research, AlAzhar University, Cairo, Egypt and the study began from March until September 2019. Inclusion criteria comprised body mass index (BMI) $<35 \mathrm{~kg} / \mathrm{m}^{2}$ and $>25$ $\mathrm{kg} / \mathrm{m}^{2}$ in the control group and $>35 \mathrm{~kg} / \mathrm{m}^{2}$ in other groups. Male age was less than 45 years old and female partners were with less than 38 years old or reasonably infertile with ICSI and free of medical disorder but suffer from the absence of a fallopian tube or no reason non-pregnant.

Males in control group had standard quality of sperms. Exclusion criteria comprised patients with aspermia, oligozoospermia, Klinefelter syndrome and with a history of cryptorchidism. Body mass index $(\mathrm{BMI})<25 \mathrm{~kg} / \mathrm{m}^{2}$ and age more than 45 years old. Patients on oral hormonal therapy and patients on weight loss programs.

Study procedures: Personal history, urogenital examination, blood analysis: hormone (FSH, $\mathrm{LH}$ and testosterone ) random glucose level, glycated hemoglobin (HbA1C), the liver function tests (aspartate aminotransferase (AST), alanine aminotransferase (ALT)), kidney function test (Urea, Creatinine) and, lipid profile (cholesterol, triglycerides, low-density lipoproteins (LDL), high-density lipoproteins (HDL) and LDL/HDL Ratio). The seminal fluid analysis was performed according to WHO 2010 criteria ${ }^{(16)}$.

Parameters measured comprised semen volume, sperm count and normal sperm morphology. Sperm preparation: Sperm samples for ICSI processed after microscopic examination, $1 \mathrm{ml}$ of sperm gradient medium were added on the fresh piece and centrifuged at $1800 \mathrm{rpm}$ for 10 minutes, the supernatant had removed, and then we add $2 \mathrm{ml}$ of sperm washing to sperms in the resulting pellet and centrifuged at 1800 rpm for 10 minutes to reach to a number of motile and morphologically normal sperm cells needed for assisted reproduction ${ }^{(16)}$.

Injected oocytes was examined for integrity and fertilization about 16-18 hours after ICSI. Oocytes was considered normally fertilized when evaluating the presence of two pronuclei (2PN) and the extrusion of the second $\mathrm{PB}$.

The Fertilization rate (FR) was calculated as the percentage transformation of microinjected oocytes into two pronuclei. Post-fertilization, about $90 \%$ of 2 $\mathrm{PN}$ oocytes obtained by ICSI enter cleavage, result in multicellular embryos. After 48 hours, zygotes that had cleaved to the two or four cell-stage, then 2-3 embryos from the best quality for each patient were transferred to the uterus of their mother. Patients performed a serum beta HCG titre $15 \pm 2$ days after oocyte collection for determination of pregnancy.

\section{Ethical approval:}

All the experimental procedures were carried out according to the principles and guidelines of the Ethics Committee of the of Faculty of science, AlAzhar University, Cairo, Egypt conformed to "Guide for the care and use of Laboratory Animals" for the use and welfare of experimental animals, published by the US National Institutes of Health (NIH publication No. 85-23, 1996).

\section{Statistical analysis}

All analyses were done using the Statistical Package for the Social Sciences version 20 for Windows (SPSS Inc, Chicago, IL). Quantitative variables were described as means $\pm \mathrm{SD}$ and were compared with the control group by an independent $\mathrm{t}$ test. Nominal and frequency data were analyzed using the Chi-square test. $\mathrm{P}$ values less than 0.05 were considered statistically significant.

\section{RESULTS}

The results in table 1 are showing the effect of physiological and biochemical disturbances in obese cases and smokers obese on fertility parameters. Where, in the OW group, the mean of liver functions ALT and AST were statistically increased compared to the normal range. According to the lipid profile analysis, the mean of the triglycerides, total cholesterol, LDL, HDL levels and LDL/HDL ratio showed a highly significant increase when comparison with the control group. Thus the mean random blood glucose was recorded a highly significant increase when comparison with the control group, but the $\mathrm{Hb} \mathrm{AIC} \%$ value was in the normal range. Also, in table 1, the kidney functions analysis in the OW group don't record any significant effect on the fertility parameters. This study shows in table 1 that people who suffer from obesity and smoking (OW\&S group) were injured the most from infertility symptoms than those who suffer from obesity only (OW group). The results showed that the kidneys' efficiency decreased with the excretion of toxic materials of creatinine and urea in the blood. It was exceeding the standard limit, and this did not happen in the case of obesity alone. Also, the liver functions were significantly decreased and give indirect effects on the fertility parameters. The mean values of ALT and AST were more than the standard control group. On the other hand, the mean random Bl Glucose level in the OW\&S was a highly significant increase than in the control group. But the mean of HbA1C per cent was average. The changes of lipid profile levels in OW\&S the mean of the triglycerides, total cholesterol, LDL levels and LDL/HDL Ratio showed a highly significant increase, when comparison with the control group. However, in OW\&S Group the mean of HDL level was not statistically significant when comparison to the control group (table 1). 
Table (1): showing the physiological and biochemical analysis between the groups

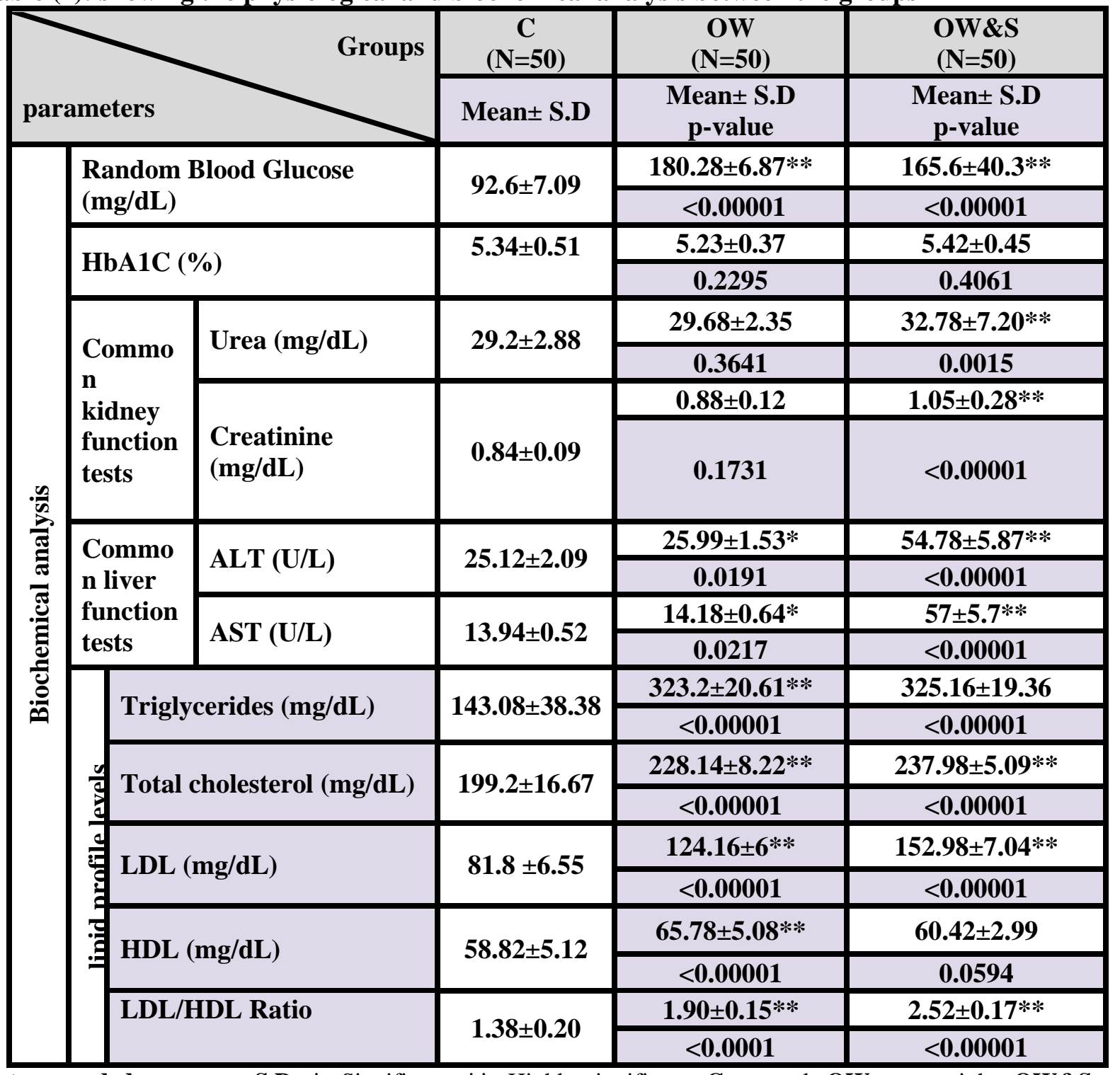

Data recorded as mean \pm S.D; *: Significant; **: Highly significant, C: control; OW: overweight; OW\&S: overweight with smoking group; AST: aspartate aminotransferase; ALT: alanine aminotransferase; HbA1C: glycated hemoglobin; LDL: low density lipoproteins; HDL: high density lipoproteins.

The parameter assessments for semen and hormonal profile are recorded in table 2. As a general view, the mean values of fertility parameters; testosterone hormone, volume of semen, sperm count, the percentage of sperm motility, were significantly decreased than the control group. However, the mean of abnormal sperm morphology percentage in the OW group, showed very highly significant increase than the control group. The mean values of FSH and LH levels showed non - significant change compared to the control values.

As showing in table 2, the sexual hormones in the overweight group with a smoking (OW\&S) showed that the mean of FSH $(13.75 \pm 2.01 \mathrm{mIU} / \mathrm{mL})$ and $\mathrm{LH}(9.3 \pm 1.98 \mathrm{mIU} / \mathrm{mL})$ hormones levels were highly significant increased $(\mathrm{P}<0.01)$ than the control group $(7.87 \pm 2.26$ and $5.02 \pm 0.69 \mathrm{mIU} / \mathrm{mL})$. However, the mean value of testosterone hormone $(2.07 \pm 0.76 \mathrm{ng} / \mathrm{dL})$ recorded a highly significant decrease $(\mathrm{P}<0.01)$ than the control value $(7.24 \pm 1.75 \mathrm{ng} / \mathrm{dL})$.

Also, table 2 showing that the outcomes of semen analysis mean in the OW\&S group recorded $2.13 \pm 0.56 \mathrm{~mL}$ semen volume, $15.98 \pm 4.72106 / \mathrm{ml}$ sperm count, and $18.9 \pm 5.28 \%$ sperm motility. These values were highly significant $(\mathrm{P}<0.01)$ decreased compared to the control group $(3.85 \pm 0.92 \mathrm{~mL}, 54.98 \pm 16.27106 / \mathrm{ml}$, and 80 $\pm 6.47 \%$, respectively). However, the mean percentage of abnormal sperm morphology in the OW\&S group was $98.22 \pm 2.84 \%$ which were a highly significant increase $(\mathrm{P}<0.01)$ than the control group $(23.5 \pm 8.16 \%)$. 
Table (2): Assessment of semen parameters and hormonal profile in the different groups **:

\begin{tabular}{|c|c|c|c|c|}
\hline \multicolumn{2}{|c|}{$\begin{array}{ll}\text { Parameter } & \text { Groups }\end{array}$} & \multirow{2}{*}{$\frac{\mathrm{C}(\mathrm{N}=50)}{\text { Mean } \pm \text { S.D }}$} & \multirow{2}{*}{$\begin{array}{c}\text { OW }(\mathrm{N}=50) \\
\begin{array}{c}\text { Mean } \pm \text { S.D } \\
\text { p-value }\end{array}\end{array}$} & \multirow{2}{*}{$\begin{array}{c}\text { OW\&S }(\mathrm{N}=50) \\
\begin{array}{c}\text { Mean } \pm \text { S.D } \\
\text { p-value }\end{array}\end{array}$} \\
\hline \multirow{7}{*}{ 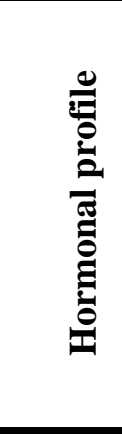 } & \multirow{3}{*}{ FSH (mIU/mL) } & & & \\
\hline & & \multirow{2}{*}{$7.87 \pm 2.26$} & $7.33 \pm 1.40$ & $13.75 \pm 02.01 * *$ \\
\hline & & & 0.172513 & $<0.00001$ \\
\hline & \multirow{2}{*}{$\mathbf{L H}(\mathbf{m I U} / \mathbf{m L})$} & \multirow{2}{*}{$5.02 \pm 0.69$} & $5.33 \pm 1.60$ & $9.3 \pm 1.98 * *$ \\
\hline & & & 0.108844 & $<0.00001$ \\
\hline & \multirow{2}{*}{$\begin{array}{l}\text { Testosterone } \\
(\text { ng/dL) }\end{array}$} & \multirow{2}{*}{$7.24 \pm 1.75$} & $2.58 \pm 0.81 * *$ & $2.07 \pm 0.76 * *$ \\
\hline & & & $<0.00001$ & $<0.00001$ \\
\hline \multirow{8}{*}{ 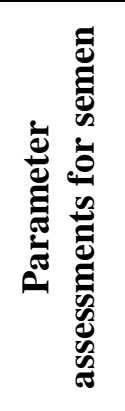 } & \multirow{2}{*}{ Volume (mL) } & \multirow{2}{*}{$3.85 \pm 0.92$} & $2.63 \pm 0.53 * *$ & $2.13 \pm 0.56 * *$ \\
\hline & & & $<0.00001$ & $<0.00001$ \\
\hline & \multirow{2}{*}{$\begin{array}{c}\text { Sperm count } \\
\left(10^{6} / \text { suspension }\right)\end{array}$} & \multirow[b]{2}{*}{$54.98 \pm 16.27$} & $33.98 \pm 5.00 * *$ & $15.98 \pm 4.72 * *$ \\
\hline & & & $<0.00001$ & $<0.00001$ \\
\hline & \multirow{2}{*}{ Sperm motility (\%) } & \multirow{2}{*}{$80 \pm 6.47$} & $36 \pm 7.69 * *$ & $18.9 \pm 4.28 * *$ \\
\hline & & & $<0.00001$ & $<0.00001$ \\
\hline & \multirow{2}{*}{$\begin{array}{l}\text { Abnormal sperm } \\
\text { morphology (\%) }\end{array}$} & \multirow{2}{*}{$23.5 \pm 8.16$} & $90.84 \pm 11.77 * *$ & $98.22 \pm 2.84 * *$ \\
\hline & & & $<0.00001$ & $<0.00001$ \\
\hline
\end{tabular}

Highly significant; $\mathbf{C}$ : control; OW: overweight; OW\&S: overweight with smoking group; $\mathbf{N}$ : number; LH: luteinizing hormone; FSH: follicle stimulating hormone.

Table 3 showing that in the overweight with smoking (OW\&S) and overweight groups (OW), the percentage of the fertilization rate and the high-quality embryos showed very highly significant decrease than the values of the control group. After the fertilization, the zygotes were successfully cleaved in all groups (100\%). In comparison, the percentage of the low-quality embryos was higher in the two groups, OW\&S, than in the control group. On the other hand, the lowest percentage of positive pregnancy test recorded in overweight with smoking followed by overweight.

Table (3): Assessment rates of fertilization, cleavage, pregnancy and embryo quality for all groups using the chisquare test

\begin{tabular}{|c|c|c|c|c|}
\hline Parameter $\quad$ Groups & $\underset{(\mathbf{N}=\mathbf{5 0})}{\mathrm{C}}$ & $\begin{array}{c}\text { OW } \\
(\mathbf{N}=\mathbf{5 0})\end{array}$ & $\begin{array}{l}\text { OW\&S } \\
(\mathrm{N}=50)\end{array}$ & $\begin{array}{l}\text { P-value of chi } \\
\text { square test }\end{array}$ \\
\hline \multirow{3}{*}{ Fertilization rate } & $\mathbf{N}$ & $\mathbf{N}$ & $\mathbf{N}$ & \multirow{3}{*}{$<0.00001 * *$} \\
\hline & 48 & 34 & 25 & \\
\hline & 96 & 68 & 50 & \\
\hline \multirow{2}{*}{$\begin{array}{l}\text { Cleavage rate at day } 3(\%) \\
\text { Fertilization) }\end{array}$} & 48 & 34 & 25 & \multirow{2}{*}{----------- } \\
\hline & 100 & 100 & 100 & \\
\hline \multirow{2}{*}{$\begin{array}{l}\text { High quality embryo (\% } \\
\text { Fertilization) }\end{array}$} & 40 & 15 & 6 & \multirow{4}{*}{$<0.00001^{* *}$} \\
\hline & 83 & 44 & 24 & \\
\hline \multirow{2}{*}{$\begin{array}{l}\text { Low quality embryo } \\
\text { (\% Fertilization) }\end{array}$} & 8 & 19 & 19 & \\
\hline & 17 & 56 & 76 & \\
\hline \multirow{2}{*}{$\begin{array}{l}\text { Positive pregnancy } \\
\text { (\% Fertilization) }\end{array}$} & 36 & 22 & 10 & \multirow{4}{*}{$<0.013^{*}$} \\
\hline & 75 & 65 & 40 & \\
\hline \multirow{2}{*}{$\begin{array}{l}\text { Negative pregnancy } \\
\text { (\% Fertilization) }\end{array}$} & 12 & 12 & 15 & \\
\hline & 25 & 35 & 60 & \\
\hline
\end{tabular}

*: Significant, **: Highly significant, C: control; OW: overweight; OW\&S: overweight with smoking group. 


\section{DISCUSSION}

Many infertile men have several problems related to sperm concentration, motility, morphology, quality and hormonal imbalance, e.g., low testosterone level. These are caused by alcoholism, drug abuse, ageing and cigarette smoking, antidepressant drugs and exposure to toxic chemicals ${ }^{(\mathbf{1 7})}$.

This study showed that people who suffer from obesity and smoking (OW\&S group) had the most from infertility symptoms than those who suffer from obesity only (OW group). The results showed that the kidneys' efficiency decreased as the excretion of toxic materials of creatinine and urea in the blood exceeded the standard limit, which did not happen in the case of obesity alone. Also, the liver functions were remarkably decreased and gave indirect effects on the fertility parameters.

In standard health cases, the liver can be cleansing of blood, vitamin synthesis, regulation of body fuel supply, cholesterol regulation, balancing hormone regulation, and control in drug metabolism. Besides, the liver plays a role in lipid metabolism by the production of low-density lipoproteins (LDL), highdensity lipoproteins (HDL) and lipid storing in itself and adipose tissues ${ }^{(18)}$. In sick cases, the increase in total cholesterol and triglyceride increases health risks, whereas a decrease in high-density lipoprotein cholesterol increases future health risk ${ }^{(\mathbf{1 9})}$.

In the present study, the mean values of fertility parameters (the testosterone hormone, the volume of semen, the sperm count and the percentage of sperm motility) significantly decreased than the control group. But, the mean of abnormal sperm morphology percentage in the OW group showed a highly significant increase than the control group. In contrast, the mean values of FSH and LH levels showed non-significant difference compared to the control values.

It was shown that paternal BMI correlated with a decrease in testosterone and an increase in estrogen levels. This hormone imbalance interferes with gonadotropin-releasing hormone $(\mathrm{GnRH}){ }^{(20)}$, and this condition may decrease sperm production (21). The secretion of accessory glands also changes with increasing BMI. In obese men, plasma seminal alphaglucosidase levels change, indicating that epididymal function has changed (22). Increasing paternal BMI reduces blastocyst development, pregnancy rate, and live birth outcomes and increases abortion rates ${ }^{(\mathbf{1 2})}$. The number of sperm in men who are overweight or obese decreases and obesity may be associated with a reduction in sperm concentration ${ }^{(9,23)}$. Paternal BMI is negatively related to the number of motile sperms, which may be due to the different classification of BMI groups between studies ${ }^{(24)}$. One study found that the effect of paternal BMI on sperm motility was only observed in severely obese men ${ }^{(\mathbf{1 0})}$. Most studies did not report the association between obesity and sperm morphology $(\mathbf{9 , 2 0 , 2 5 , 2 6 )}$.

The sexual hormones in the group of overweight with a smoking $(\mathrm{OW} \& \mathrm{~S})$ showed that the mean of FSH and LH hormones levels were highly significantly increased than the control group. However, the mean value of testosterone hormone decreased compared to the control value. It recorded a highly significant decrease than the control group. Zhao et al.. (27) in a meta-analysis of 13317 men, where it was found that smoking was associated with higher mean testosterone levels, and this was attributed to the inhibition of testosterone breakdown by cotinine.

The outcomes of semen analysis (volume, sperm count, and sperm motility) in the OW\&S group recorded high significant decrease compared to the control group. Thus, the mean percentage of abnormal sperm morphology in the OW\&S group was significantly higher than in the control group. Besides its association with impaired male fertility, tobacco smoking is also responsible for increases in DNA damage, aneuploidies, and mutations in sperm ${ }^{(28)}$.

In the present study, the percentage of the fertilization rate and the rate of high-quality embryos was significantly decreased in the overweight with smoking (OW\&S) and overweight groups (OW) than the values of the control group. After the fertilization, the zygotes were successfully cleaved in all groups $(100 \%)$. But the percentage of the low-quality embryo was higher in OW\&S and OW groups than in the control group. On the other hand, the lowest rate of positive pregnancy test recorded in overweight with smoking (40\%) followed by the overweight group (65\%) compared to the positive and negative pregnancy rates in the control group (75\% and $25 \%$ ).

Further, male obesity and raised male BMI can markedly harm quality embryo blastocyst development, clinical pregnancy rate, and live birth rate in couples who underwent IVF or ICSI ${ }^{(12,29-31)}$. No correlation was found between paternal BMI and embryo development until the third day. The paternal genome is inactivated up to the four- to the eight-cell stage in human embryos, so the effect of paternal obesity on sperm integrity and function is limited before this stage ${ }^{(\mathbf{1 0})}$.

With paternal smoking being a significant risk factor for in vitro fertilization (IVF) and intracytoplasmic sperm injection (ICSI) failure, paternal smoking could perhaps contribute to decreased assisted reproductive technology (ART) success rates as much as maternal smoking (a risk factor only for IVF failure) does or more ${ }^{(32)}$. Moreover, male smoking could even influence the clinical pregnancy rate per intrauterine insemination (IUI) cycle ${ }^{(33)}$. Amongst former smokers, every additional year following the male partner's smoking cessation reduced the risk of ART failure by $4 \%$, particularly between clinical pregnancy and lived birth $^{(34)}$.

\section{CONCLUSION}

Male overweight with or without smoking had a significant negative impact on semen parameters and hormonal profile, fertilization, embryo quality and pregnancy rate. 


\section{ACKNOWLEDGEMENT}

We are grateful to all the team members in the Assisted Reproductive Unit in International Islamic Center for Population Studies and Research, Al-Azhar University.

\section{REFERENCES}

1. Tajaddini Mahani S, Behnam B, Abbassi M et al. (2016): Tsga10 expression correlates with sperm profiles in the adult formalin-exposed mice. Andrologia, 48 (10):1092-1101.

2. Kumar N, Singh A (2015): Trends of male factor infertility, an important cause of infertility: a review of literature. $\mathbf{J}$ Hum Reprod Sci., 8(4):191-198.

3. Zeqiraj A, Beadini S, Beadini $N$ et al. (2018): Male infertility and sperm DNA fragmentation. Open Access Maced J Med Sci., 6(8):1342-1347.

4. Zini A, Jamal W, Cowan L et al. (2011): Is sperm DNA damage associated with IVF embryo quality? A systematic review. J Assist Reprod Genet., 28(5):391-399.

5. Sadeghi M, Hodjat M, Lakpour N et al. (2009): Effects of sperm chromatin integrity on fertilization rate and embryo quality following intracytoplasmic sperm injection. Avicenna J Med Biotechnol.. 1(3):173.

6. Haslam D, James W (2005): Obesity. Lancet, 366:11971209.

7. World Health Organization (2000): Obesity: preventing and managing the global epidemic. Geneva: WHO Technical Report Series number 894. https://apps.who.int/iris/handle/10665/42330

8. Begg S, Vos T, Barker B et al. (2008): Burden of disease and injury in Australia in the new millennium: measuring health loss from diseases, injuries and risk factors. Med J Aust., 188(1):36-40.

9. Jensen T, Andersson A, Jørgensen N et al. (2004): Body mass index in relation to semen quality and reproductive hormonesamong 1,558 Danish men. Fertil Steril., 82(4):863-70.

10. Bakos H, Henshaw R, Mitchell M et al. (2011): Paternal body mass index is associated with decreased blastocyst development and reduced live birth rates following assisted reproductive technology. Fertil Steril.,95(5):1700-1704.

11. Yang Q, Zhao F, Hu L et al. (2011): Effect of paternal overweight or obesity on IVF treatment outcomes and the possible mechanisms involved. Sci Rep., 6:29787-29793.

12. Tola E, Oral H (2019): The role of paternal obesity on the success of intracytoplasmic sperm injection cycle a tertiary IVF center in Turkey. JPMA J Pak Med Assoc., 69(5):6406.

13. Fims S, Cruzat V, Keane $K$ et al. (2015): The effect of cigarette smoking, alcohol consumption and fruit and vegetable consumption on IVF outcomes: A review and presentation of original data. Reprod Biol Endocrinol., 13:134-139.

14. Oliveira H, Spanò M, Santos C et al. (2011): Adverse effects of cadmium exposure on mouse sperm. Reprod Toxicol., 28(4):550-555.

15. Lanzafame M, La Vignera S, Vicari E et al. (2009): Oxidative stress and medical antioxidant treatment in male infertility. Reprod Biomed Online, 19(5):638-659.

16. Catanzariti F, Cantoro $U$, Lacetera $V$ et al. (2013): Comparison between WHO (World Health Organization) 2010 and WHO 1999 parameters for semen analysis interpretation of 529 consecutive samples. Arch Ital di Urol e Androl., 85 (3):125-128.
17. Kahn B, Brannigan R (2017): Obesity and male infertility. Current Opinion in Urology, 27(5):441-445.

18. Cohen D, Corey $K$ (2019): Lipid and lipoprotein metabolism in liver disease. In: De Groot LJ, Beck-Peccoz P, Chrousos G, Dungan K, Grossman A, Hershman JM, Koch C, McLachlan R, New M, Rebar R, Singer F, Vinik A, Weickert MO, eds. Endotext. South Dartmouth (MA) 2019. https://www.ncbi.nlm.nih.gov/books/NBK395573/

19. Mondal S, Mukhopadhyay S (2018): Effect of central obesity on lipid profile in healthy young adults. Medical Journal of Dr. DY Patil Vidyapeeth., 11(2): 152-158.

20. Chavarro J, Toth T, Wright $D$ et al. (2010): Body mass index in relation to semen quality, sperm DNA integrity, and serum reproductive hormone levels among men attending an infertility clinic. Fertil Steril., 93(7):2222-2253.

21. Vilarino F, Christofolini D, Rodrigues D et al. (2011): Body mass index and fertility: is there a correlation with human reproduction outcomes? Gynecol Endocrinol., 27(4):232-238

22. Raad G, Hazzouri M, Bottini S et al. (2017): Paternal obesity: how bad is it for sperm quality and progeny health? Basic Clin Androl., 27(1):20-32.

23. Fejes I, Koloszar S, Szöllo" si J et al. (2005): Is semen quality affected by male body fat distribution? Andrologia, 37(5):155-164.

24. Kort H, Massey J, Elsner C et al. (2006): Impact of body mass index values on sperm quantity and quality. J Androl., 27(3):450-453.

25. Fejes I, Závaczki Z, Szöllősi J et al. (2005): Is there a relationship between cell phone use and semen quality? Arch Androl., 51(5):385-393.

26. Pauli E, Legro R, Kunselman A et al. (2008): Diminished paternity and gonadal function with increasing obesity in men. Fertil Steril., 90 (2):346-351.

27. Zhao J, Leung J, Lin $\mathbf{S}$ et al. (2016): Cigarette smoking and testosterone in men and women: A systematic review and meta-analysis of observational studies. Prev Med., 85:1-10.

28. Beal M, Yauk C, Marchetti F (2017): From sperm to offspring: Assessing the heritable genetic consequences of paternal smoking and potential public health impacts. Mutat Res., 773:26-50.

29. Davidson LM, Millar K, Jones C et al. (2015): Deleterious effects of obesity upon the hormonal and molecular mechanisms controlling spermatogenesis and male fertility, Hum. Fertil., 18 (3):184-193.

30. Le W, Su S, Shi L et al. (2016): Effect of male body mass index on clinical outcomes following assisted reproductive technology: a meta-analysis. Andrologia, 48(4): 406-424.

31. Mushtaq R, Pundir J, Achilli C et al. (2018): Effect of male body mass index on assisted reproduction treatment outcome: an updated systematic review and metaanalysis. Reproductive Biomedicine Online, 36(4): 459471.

32. Kovac J, Khanna A, Lipshultz L (2015): The effects of cigarette smoking on male fertility. Postgrad Med., 127:338-341.

33. Thijssen A, Creemers A, Van der Elst W et al. (2017): Predictive value of different covariates influencing pregnancy rate following intrauterine insemination with homologous semen: a prospective cohort study. Reprod Biomed Online, 34:463-472.

34. Vanegas J, Chavarro J, Williams P et al. (2017): Discrete survival model analysis of a couple's smoking pattern and outcomes of assisted reproduction. Fertil Res Pract., 3:5-15. 